\title{
A Comparison of Blood Pressure Variability Assessed over Varying Time Periods using Different Methods of Measurement in Patients with Cerebrovascular Disease
}

\author{
Davison WJ, Myint PK, Potter JF.
}

\section{Introduction}

Blood pressure variability (BPV) can be measured over different timescales with beat-to beat blood pressure (BP) data showing variability over minutes, ambulatory BP monitoring (ABPM) variability over hours, and self-BP monitoring (SBPM) variability over days. Long-term BPV is possibly a better predictor of cardiovascular risk than short-term BPV ${ }^{1}$ and this may relate to differing underlying pathophysiological mechanisms. ${ }^{2-4}$ Consequently, whether variability over different timescales is a singular phenomenon is unclear.

\section{Methods}

- Adults with TIA/stroke (NIHSS $<15)$ were recruited between 72 hours and 12 weeks post-event.

- All participants had 10 minutes of beat-to-beat BP monitoring (Task Force ${ }^{\circledR}$ Monitor), at rest in the supine position ( $\geq 5$ minutes valid recording) and daytime ambulatory BP monitoring ((ABPM) SpaceLabs 90207) with BP measured at 20 minute intervals ( $\geq 14$ readings).

- A subset also completed self-BP monitoring (SBPM) at home over 7 days (Omron 705IT), taking 2 readings every morning and evening, seated at rest, before meals and antihypertensive medications ( $\geq 21$ readings).

- For each set of measurements BPV of systolic BP (SBP) and diastolic BP (DBP) were calculated as the standard deviation (SD), coefficient of variation ((CV) $100 x$ $\mathrm{SD} /$ mean), average real variability ((ARV) mean of the absolute difference between successive BP measurements), maximum, and maximum-minimum difference ((MMD) maximum BP - minimum BP).

- Data were analysed using SPSS (v.23.0). Values for each BPV index were compared across measurement methods using Pearson's correlation coefficient. Participants were divided into quartiles for each index and the agreement of classification by quartile was assessed using the kappa statistic.

\section{Results}

- 158 participants with 95 in the SBPM subset.

- Correlations for mean BP between methods were all significant.

- Correlations for BPV indices between methods were mostly weak or non-significant.

- Agreement of classification by quartiles was weak or nonsignificant for all variability indices (data not shown).

\begin{tabular}{|l|l|}
\hline Age (years) & $73.6(10.0)$ \\
\hline Sex (male) & $104(66 \%)$ \\
\hline Diagnosis (TIA) & $106(67 \%)$ \\
\hline Time to recruitment (weeks) & $9.1(3.2)$ \\
\hline Clinic SBP $(\mathrm{mmHg})$ & $151.6(19.4)$ \\
\hline Clinic DBP $(\mathrm{mmHg})$ & $83.7(11.9)$ \\
\hline Antihypertensive treatment & $147(93 \%)$ \\
\hline
\end{tabular}

\begin{tabular}{|c|c|c|c|c|}
\hline & & $\begin{array}{c}\text { Beat-to-beat } \\
(\mathrm{N}=138)\end{array}$ & $\begin{array}{c}\text { ABPM } \\
(\mathrm{N}=154)\end{array}$ & $\begin{array}{l}\text { SBPM } \\
(\mathrm{N}=95)\end{array}$ \\
\hline \multirow{2}{*}{$\begin{array}{c}\text { Mean } \\
(\mathrm{mmHg})\end{array}$} & SBP & $131.6(16.2)$ & $134.5(14.2)$ & $141.1(15.6)$ \\
\hline & DBP & $80.8(12.6)$ & $76.0(8.9)$ & $78.1(8.9)$ \\
\hline \multirow{2}{*}{$\begin{array}{c}\mathrm{SD} \\
(\mathrm{mmHg})\end{array}$} & SBP & $6.2(3.4)$ & 13.5 (3.4) & $11.6(3.2)$ \\
\hline & DBP & $4.7(2.6)$ & $9.2(2.4)$ & $6.6(2.1)$ \\
\hline \multirow{2}{*}{ CV (\%) } & SBP & $4.7(2.5)$ & $10.1(2.6)$ & $8.3(2.1)$ \\
\hline & DBP & $5.9(3.5)$ & $12.2(3.5)$ & $8.5(2.7)$ \\
\hline \multirow{2}{*}{$\begin{array}{c}\text { ARV } \\
(\mathrm{mmHg})\end{array}$} & SBP & $1.9(1.2)$ & $9.7(2.8)$ & $11.6(3.6)$ \\
\hline & DBP & $1.5(1.2)$ & $7.3(1.8)$ & $6.4(2.7)$ \\
\hline \multirow{2}{*}{$\begin{array}{c}\mathrm{Max} \\
(\mathrm{mmHg})\end{array}$} & SBP & 150.0 & $166.3(18.0)$ & $164.8(18.5)$ \\
\hline & DBP & $97.0(16.4)$ & $99.0(11.9)$ & $92.9(11.9)$ \\
\hline \multirow{2}{*}{$\begin{array}{c}\text { MMD } \\
(\mathrm{mmHg})\end{array}$} & SBP & $39.3(20.7)$ & $59.8(16.4)$ & $46.7(13.8)$ \\
\hline & DBP & $30.7(14.6)$ & $47.5(12.0)$ & $27.2(10.3)$ \\
\hline
\end{tabular}

Table 2: Mean and BPV values from each BP measurement method. Data presented are mean (SD).

\begin{tabular}{|c|c|c|c|c|}
\hline & $\begin{array}{c}\text { Beat-to-beat } \\
\text { vs. ABPM } \\
(\mathrm{N}=134)\end{array}$ & $\begin{array}{c}\text { Beat-to-beat } \\
\text { vs. SBPM } \\
\text { (N=93) }\end{array}$ & $\begin{array}{c}\text { ABPM vs } \\
\text { SBPM } \\
\text { (N=93) }\end{array}$ \\
\hline \multirow{2}{*}{ Mean } & SBP & $0.35^{\dagger}$ & $0.50^{\dagger}$ & $0.55^{\dagger}$ \\
\hline & DBP & $0.57^{\dagger}$ & $0.57^{\dagger}$ & $0.53^{\dagger}$ \\
\hline \multirow{2}{*}{ SD } & SBP & $0.26^{*}$ & 0.12 & $0.32^{\dagger}$ \\
\hline & DBP & $0.20^{*}$ & 0.11 & 0.16 \\
\hline \multirow{2}{*}{ CV } & SBP & $0.19^{*}$ & 0.05 & $0.35^{\dagger}$ \\
\hline & DBP & $0.18^{*}$ & 0.12 & 0.15 \\
\hline \multirow{2}{*}{ ARV } & SBP & 0.09 & 0.19 & $0.28^{\dagger}$ \\
\hline & DBP & $0.20^{*}$ & 0.16 & 0.17 \\
\hline \multirow{2}{*}{$\operatorname{Max}$} & SBP & $0.24^{*}$ & $0.47^{\dagger}$ & $0.45^{\dagger}$ \\
\hline & DBP & $0.35^{\dagger}$ & $0.32^{\dagger}$ & 0.14 \\
\hline \multirow{2}{*}{ MMD } & SBP & $0.22^{*}$ & 0.19 & $0.25^{\star}$ \\
\hline & DBP & $0.21^{*}$ & 0.16 & 0.07 \\
\hline
\end{tabular}

Table 3: Pearson's correlation coefficients ( $r$ values) for mean $\mathrm{BP}$ and measures of BPV compared between measurement methods. ${ }^{*} p<0.05 .{ }^{\dagger} p<0.01$

Table 1: Demographics, presented as mean (SD) or count (\%).

\section{Conclusions}

- Short-term and medium-term BPV are not strongly correlated regardless of the statistical index used to calculate variability.

- Considering this with the evidence that the prognostic value and underlying mechanisms of different timescales of BPV are not consistent it is likely that short, medium, and long-term variability are not exactly the same phenomenon. 